\title{
Anti-wrinkle effects of Sargassum muticum ethyl acetate fraction on ultraviolet B-irradiated hairless mouse skin and mechanistic evaluation in the human HaCaT keratinocyte cell line
}

\author{
JAE HYOUNG SONG $^{1 *}$, MEI JING PIAO ${ }^{2 *}$, XIA HAN $^{2}$, KYOUNG AH KANG $^{2}$, HEE KYOUNG KANG ${ }^{2}$,

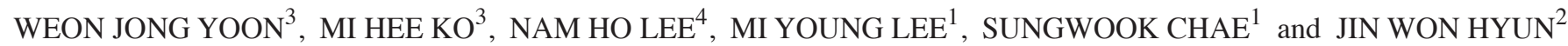 \\ ${ }^{1}$ KM Convergence Research Division, Korea Institute of Oriental Medicine, Daejeon 34054; ${ }^{2}$ School of Medicine, \\ Jeju National University, Jeju 63243; ${ }^{3}$ Jeju Biodiversity Research Institute, Jeju High-Tech Development Institute, Jeju 63612; \\ ${ }^{4}$ Department of Chemistry, College of Natural Sciences, Jeju National University, Jeju 63243, Republic of Korea
}

Received July 28, 2015; Accepted June 17, 2016

DOI: $10.3892 / \mathrm{mmr} .2016 .5655$

\begin{abstract}
The present study investigated the photoprotective properties of the ethyl acetate fraction of Sargassum muticum (SME) against ultraviolet B (UVB)-induced skin damage and photoaging in a mouse model. HR-1 strain hairless male mice were divided into three groups: An untreated control group, a UVB-irradiated vehicle group and a UVB-irradiated SME group. The UVB-irradiated mice in the SME group were orally administered with SME $(100 \mathrm{mg} / \mathrm{kg}$ body weight in $0.1 \mathrm{ml}$ water per day) and then exposed to radiation at a dose of $60-120 \mathrm{~mJ} / \mathrm{cm}^{2}$. Wrinkle formation and skin damage were evaluated by analysis of skin replicas, epidermal thickness and collagen fiber integrity in the dermal connective tissue. The mechanism underlying the action of SME was also investigated in the human $\mathrm{HaCaT}$ keratinocyte cell line following exposure of the cells to UVB at a dose of $30 \mathrm{~mJ} /$ $\mathrm{cm}^{2}$. The protein expression levels and activity of matrix metalloproteinase-1 (MMP-1), and the binding of activator protein-1 (AP-1) to the MMP-1 promoter were assessed in the HaCaT cells using western blot analysis, an MMP-1 fluorescent assay and a chromatin immune-precipitation assay, respectively. The results showed that the mean length and depth of the wrinkles in the UVB-exposed hairless mice were
\end{abstract}

Correspondence to: Professor Jin Won Hyun, School of Medicine, Jeju National University, 102 Jejudaehakro, Jeju 63243, Republic of Korea

E-mail: jinwonh@jejunu.ac.kr

Dr Sungwook Chae, KM Convergence Research Division, Korea Institute of Oriental Medicine, 1672 Yuseong-daero, Yuseong-gu, Daejeon 34054, Republic of Korea

E-mail: kendall@kiom.re.kr

${ }^{*}$ Contributed equally

Key words: collagen, matrix metalloproteinase-1, skin photoaging, Sargassum muticum, skin wrinkling, ultraviolet B significantly improved by oral administration of SME, which also prevented the increase in epidermal thickness triggered by UVB irradiation. Furthermore, a marked increase in collagen bundle formation was observed in the UVB-treated mice with SME administration. SME pretreatment also significantly inhibited the UVB-induced upregulation in the expression and activity of MMP-1 in the cultured HaCaT keratinocytes, and the UVB-enhanced association of AP-1 with the MMP-1 promoter. These results suggested that SME may be useful as an anti-photoaging resource for the skin.

\section{Introduction}

Previous studies have demonstrated that the oral ingestion of phytochemicals found in herbs, vegetables and fruits can safeguard the skin against ultraviolet B (UVB)-induced photodamage (1-3). The condition and appearance of the irradiated skin are improved by dietary supplementation with phytochemicals. For example, Honeybush (Cyclopia) is rich in antioxidant polyphenols, and these compounds can protect SKH-1 strain hairless mice from UVB-triggered skin injury (4). In addition, the oral administration of antioxidant proanthocyanidins and polyphenols derived from grape seeds and green tea, respectively, can prevent UVB-associated photocarcinogenesis and oxidative skin damage in SKH-1 mice $(5,6)$. These and other antioxidants are essential as scavengers of the intracellular reactive oxygen species (ROS), the levels of which are elevated by exposure of the skin to UVB radiation, and include singlet oxygen, superoxide anions, hydroxyl radicals and hydrogen peroxide (7). The functions of ROS have been investigated for their contribution to photoaging, inflammation and numerous disease processes (8-10).

UV exposure frequently provokes skin pigmentation and wrinkling, and also stimulates the expression and activity of matrix metalloproteinases (MMPs). The latter degrade all types of extracellular matrix proteins, thereby damaging connective tissues. Collagen is the most abundant protein in the dermal connective tissue, and is enzymatically digested by MMP-1 or interstitial collagenase. Collagen degradation and damage feature predominantly in photoaging (11), and thus an 
increase in the expression and/or activity of MMP-1 is a hallmark of UV-mediated skin injury. The exogenous production of free radicals via ionizing UV radiation further damages the skin at cellular and tissue levels. Although the body possesses a defense system to protect against this oxidative damage, excessive levels of free radicals and ROS can overwhelm this defense system and incite a state of oxidative stress or immunosuppression, and potential carcinogenesis. However, antioxidant supplementation provides additional protection to neutralize ROS (12).

The brown alga, Sargassum muticum, is widely distributed on the coastline of the southern and eastern areas of Korea. Extracts derived from $S$. muticum reportedly demonstrate antioxidant, antimicrobial and anti-inflammatory activities $(13,14)$. In previous screening for anti-photoaging candidates, it was found that an ethyl acetate extract of S. muticum (SME) exerted cytoprotective activity against UVB irradiation in cultured human $\mathrm{HaCaT}$ keratinocytes by scavenging free radicals and inducing the expression of antioxidant enzymes (15). However, to the best of our knowledge, no previous studies have been performed to investigate the ability of SME to defend against UVB-induced skin aging in an animal model. Therefore, the present study examined the capacity of SME to safeguard HR-1 strain hairless mice against UVB-provoked photoaging, oxidative stress and wrinkling, and investigated the potential mechanism underling the action of SME in the HaCaT cell line. The in vivo and in vitro results suggest SME as a potential candidate for clinical trials and drug development. Further study is required in order to fully elucidate the use of SME in humans.

\section{Materials and methods}

Preparation of the SME. Samples of S. muticum were collected from Udo (Jeju Island, Korea) and identified by Dr Dong Sam Kim (Jeju Biodiversity Research Institute, Jeju, Republic of Korea). A voucher specimen (A10-0000107) was deposited at the herbarium of Jeju Biodiversity Research Institute (Jeju, Korea). S. muticum was extracted with $80 \%$ ethanol under reflux. Subsequently, the $80 \%$ ethanol extract was suspended in distilled water and fractionated successively with $n$-hexane, dichloromethane, ethyl acetate and $n$-butanol (Merck Millipore, Darmstadt, Germany). The ethyl vacetate fraction of $S$. muticum (SME) was used for further experiments.

Reagents. The primary antibody against MMP-1 was purchased from Epitomics (Burlingame, CA, USA), and the primary antibody against actin was obtained from Sigma-Aldrich (St. Louis, MO, USA). The primary antibodies against c-Jun and phosphorylated (phospho)-c-Jun were purchased from Cell Signaling Technology, Inc. (Danvers, MA, USA). All other chemicals and reagents were of analytical grade, unless otherwise stated.

Experimental animals, oral administration of SME and $U V B$ radiation. HR-1 strain hairless male mice (6 weeks old; 22-24 g) were purchased from Japan SLC, Inc. (Shizuoka, Japan) and allowed to acclimate to conditions in the facility for 1 week prior to experimentation. The animals were housed in climate-controlled quarters $\left(24^{\circ} \mathrm{C}\right.$ at $50 \%$ humidity) with a $12 / 12 \mathrm{~h}$ light/dark cycle and free access to standard rodent chow and water. Subsequent to the 1 week acclimation period, the mice were divided into three groups: An untreated control group $(n=5)$, a UVB-treated vehicle group $(n=5)$ and a UVB-treated SME group $(n=5)$. The mice in the SME group were orally administered with SME $(100 \mathrm{mg} / \mathrm{kg}$ body weight in $0.1 \mathrm{ml}$ of water per day). SME was administered 5 days/week for 12 weeks. Exposure to UVB irradiation was then performed using an UVM-225D Mineralight UV display lamp (UVP, Inc., Phoenix, AZ, USA) emitting UV light at a wavelength of $302 \mathrm{~nm}$. The UV strength was measured using a HD2102-2 UV meter (Delta OHM, Padova, Italy). UVB radiation was applied to the skin on the backs of the animals three times per week for 12 weeks, with the UVB dose progressively increased between $60 \mathrm{~mJ} / \mathrm{cm}^{2}$ per exposure in week 1 (one minimal erythematous dose $=60 \mathrm{~mJ} / \mathrm{cm}^{2}$ ) and $120 \mathrm{~mJ} / \mathrm{cm}^{2}$ per exposure in week 7 . The experimental protocol was approved by the Institutional Animal Care and Use Committee of the Korea Institute of Oriental Medicine (Daejeon, Korea; approval no. 11-061). All experimental protocols were approved by the Korea Institute of Oriental Medicine Institutional Animal Care and Use Committee (11-061).

Cell culture and $U V B$ radiation. The HaCaT keratinocyte cell line was obtained from Amore Pacific Company (Yongin, Korea). The cells were maintained at $37^{\circ} \mathrm{C}$ in an incubator with a humidified atmosphere of $5 \% \mathrm{CO}_{2}$, and were cultured in Dulbecco's modified Eagle's medium (Gibco; Thermo Fisher Scientific, Inc., Waltham, MA, USA) containing $10 \%$ heat-inactivated fetal calf serum (Gibco; Thermo Fisher Scientific, Inc.), streptomycin $(100 \mu \mathrm{g} / \mathrm{ml})$ and penicillin $(100 \mathrm{U} / \mathrm{ml})$. For irradiation, the cells were exposed to UVB light once at a dose of $30 \mathrm{~mJ} / \mathrm{cm}^{2}$. The CL-1000 M UV Crosslinker (UVP, Inc.) was used as the UVB source, which delivered a UVB energy spectrum of 280-320 nm.

Generation of skin replicas and image analysis. Mice were anesthetized with intraperitoneal administration of a diluted solution (1:4 in phosphate-buffered saline) composed of a 2:1 mixture of $30 \mathrm{mg} / \mathrm{kg}$ Zoletil (Virbac, Carros, France) and $10 \mathrm{mg} / \mathrm{kg}$ Rompun (Bayer, Leverkusen, Germany) at the end of final oral administration. Mice were sacrificed using a $\mathrm{CO}_{2}$ chamber subsequent to the final oral administration. Mouse dorsal skin replicas were obtained using a Repliflo Cartridge kit (CuDerm Corporation, Dallas, TX, USA). An image of the dorsal skin of each mouse was captured prior to the sacrifice of the animal. The impression replicas were placed on a horizontal sample stand, and wrinkle shadows were generated by illumination using a fixed-intensity optical light at an angle of $35^{\circ}$. Black and white images were recorded using a charge-coupled device camera and were analyzed using Skin-Visiometer ${ }^{\circledR}$ VL 650 software (Courage + Khazaka Electronic GmbH, Cologne, Germany). The average wrinkle lengths and depths were measured, and used as parameters to assess skin wrinkling.

Histological examination of the skin. The thickness of the epidermis was measured via light microscopy using an eyepiece micrometer (AX-70; Olympus Corporation, Tokyo, Japan). For 
this analysis, the dorsal skin ( $2 \times 2 \mathrm{~cm}$ samples) was obtained under anesthesia and was fixed in $10 \%$ neutral-buffered formalin, embedded in paraffin and sectioned at a thickness of $5 \mu \mathrm{m}$. The sections were stained with hematoxylin and eosin to evaluate tissue morphology, or with Masson's trichrome stain for collagen fiber analysis.

Western blot analysis. The harvested HaCaT keratinocytes were lysed by incubating the cells on ice for $10 \mathrm{~min}$ in $100 \mu 1$ PRO-PREP ${ }^{\mathrm{TM}}$ protein extraction solution (Intron Biotechnology (Seongnam, Korea). The cell lysates were centrifuged at $16,000 \times \mathrm{g}$ at $4^{\circ} \mathrm{C}$ for $5 \mathrm{~min}$, and the supernatants were removed from the lysates. Protein concentrations were then determined using Quant-i $\mathrm{T}^{\mathrm{TM}}$ Protein Assay kit (Invitrogen; Thermo Fisher Scientific, Inc.). Aliquots of the lysates (40 $\mu \mathrm{g}$ of protein) were boiled for $5 \mathrm{~min}$ and electrophoresed in a $10 \%$ sodium dodecyl sulfate-polyacrylamide gel. The proteins within the gel were then transferred onto nitrocellulose membranes, and the membranes were subsequently incubated with the rabbit monoclonal antibodies against MMP-1 (cat. no. \#1973-1; 1:1,000) and phospho-c-Jun (Ser73; cat. no. D47G9; 1:1,000). Following incubation with primary antibodies, the membranes were further incubated with secondary anti-immunoglobulin G/horseradish peroxidase conjugates (Pierce Biotechnology, Inc., Rockford, IL, USA). Protein bands were visualized using an Enhanced Chemiluminescence Western Blotting Detection kit (GE Healthcare Life Sciences, Little Chalfont, UK).

Determination of MMP-1 activity. MMP-1 activity was assessed using a Fluorokine ${ }^{\circledR}$ E Human Active MMP-1 Fluorescent Assay kit (R\&D Systems, Inc., Minneapolis, MN, USA). This assay uses a quenched fluorogenic substrate to detect enzyme activity. Production of the fluorescent cleavage product was measured using a fluorescence plate reader (BMG Labtech, Ortenberg, Germany) with excitation and emission wavelengths of 320 and $405 \mathrm{~nm}$, respectively.

Chromatin immunoprecipitation (ChIP) assay. The ChIP assay was performed using a SimpleChIP ${ }^{\text {тм }}$ Enzymatic Chromatin IP kit (Cell Signaling Technology, Inc.), according to the manufacturer's protocol with slight modification. Briefly, the HaCaT keratinocytes were crosslinked by the addition of $1 \%$ formaldehyde, and the prepared chromatin was enzymatically digested with nuclease for $20 \mathrm{~min}$ at $37^{\circ} \mathrm{C}$. Primary antibody against the activator protein-1 (AP-1) family member, c-Jun rabbit monoclonal antibody (60A8; cat. no. \#9165; 1:50; Cell Signaling Technology, Inc.), and normal rabbit polyclonal IgG (cat. no. \#2729; 1:500; Cell Signaling Technology, Inc.) were added to the chromatin digest, which was then incubated overnight with constant rotation at $4^{\circ} \mathrm{C}$. To capture the immunoprecipitated DNA/ protein complexes, ChIP-grade protein $\mathrm{G}$ magnetic beads were added to the digest. Pellet protein $\mathrm{G}$ agarose was obtained by brief centrifugation $\left(5,000 \mathrm{x}\right.$ g for $1 \mathrm{~min}$ at $\left.4^{\circ} \mathrm{C}\right)$ and the supernatant fraction was removed. The protein $G$ agarose-antibody/chromatin complex was washed by resuspending the beads in $1 \mathrm{ml}$ each of the cold buffers in the order listed below, and incubation for $5 \mathrm{~min}$ on a rotating platform followed by brief centrifugation $(5,000 \mathrm{x} \mathrm{g}$ for $1 \mathrm{~min}$ at $4^{\circ} \mathrm{C}$ ) and careful removal of the supernatant fraction. The immunoprecipitates were eluted with ChIP elution buffer. The crosslinking was reversed by incubating the eluent at $65^{\circ} \mathrm{C}$ for $30 \mathrm{~min}$, followed by the addition of proteinase $\mathrm{K}$ and further incubation at $65^{\circ} \mathrm{C}$ for $2 \mathrm{~h}$.

The DNA fragments recovered from the immunoprecipitated DNA/protein complex were purified on spin columns. DNA was amplified in a reaction containing primers, dNTPs, and 0.5 U Taq DNA polymerase (Intron Biotechnology) at a final volume of $25 \mu \mathrm{l}$. The PCR conditions were: $5 \mathrm{~min}$ at $95^{\circ} \mathrm{C}$ for initial denaturation, followed by 45 cycles of $30 \mathrm{sec}$ at $94^{\circ} \mathrm{C}, 1 \mathrm{~min}$ at $49^{\circ} \mathrm{C}$ and $1 \mathrm{~min}$ at $72^{\circ} \mathrm{C}$, and a final elongation period of $7 \mathrm{~min}$ at $72^{\circ} \mathrm{C}$. PCR amplification was conducted in an Applied Biosystems 2720 thermal cycler (Applied Biosystems; Thermo Fisher Scientific, Inc.). The primers for the MMP-1 gene promoter were as follows: Sense 5'-CCTCTT GCTGCTCCAATATC-3' and antisense 5'-TCT GCTAGGAGTCACCATTTC-3' (Bioneer Corporation, Daejeon, Korea). The promoter region of the MMP-1 gene exists between -67 and +94 of the MMP-1 gene sequence, with reference to the transcription start site. The PCR products were separated on $2 \%$ agarose gels, and the DNA bands were visualized using RedSafe ${ }^{\mathrm{TM}}$ nucleic acid staining solution (Intron Biotechnology) and an ImageQuant 350 digital imaging system (GE Healthcare, Wankesha, WI, USA).

Statistical analysis. All measurements were obtained in triplicate, and all values are presented as the mean \pm the standard error of the mean. The results were subjected to two-way analysis of variance followed by the F-test using GraphPad Prism 5 (GraphPad Software, Inc., La Jolla, CA, USA) to analyze differences between conditions. In all cases, $\mathrm{P}<0.05$ was considered to indicate a statistically significant difference.

\section{Results}

SME inhibits UVB-induced wrinkle formation in mouse skin. To investigate the effects of SME on UVB-induced wrinkle formation in vivo, the skin on the backs of HR-1 hairless mice was exposed to UVB light for a period of 12 weeks. UVB exposure resulted in macroscopic wrinkle formation in the dorsal skin of the vehicle-treated mice, compared with the unexposed control mice. However, wrinkling of the UVB-irradiated skin was markedly inhibited by SME pretreatment (Fig. 1A). To further evaluate the protective actions of SME against UVB-mediated skin damage, replicas of the mouse dorsal skin were obtained (Fig. 1B). SME visibly inhibited wrinkle formation in replica images of UVB-exposed skin, consistent with the macroscopic observations. Skin replicas were further evaluated using an image analysis system to quantify the degree of wrinkle formation. The mean length and depth of the wrinkles were significantly higher in the UVB-treated vehicle group, compared with those in the unexposed control group (Fig. 2A and B). However, the mean wrinkle length and depth were significantly decreased in the UVB-treated mice pretreated with SME.

SME reduces epidermal thickness in UVB-irradiated mouse skin. As epidermal thickness is used as a quantitative parameter to assess inflammation and skin photoaging (16), the 
A

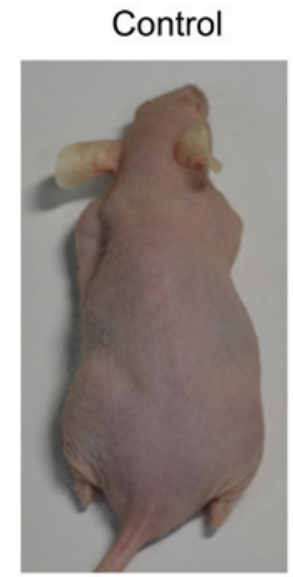

B

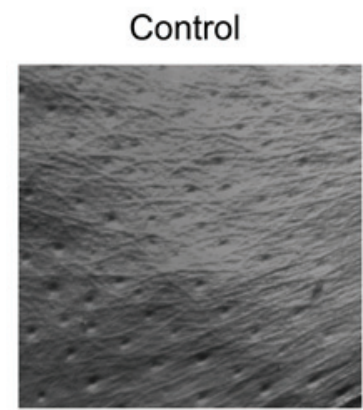

UVB

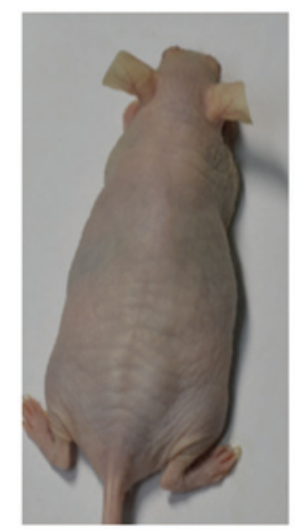

UVB

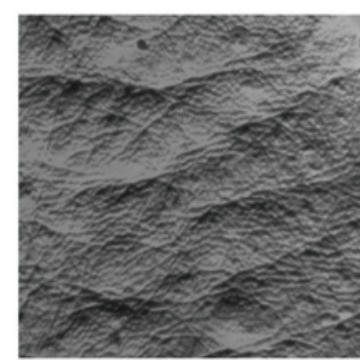

SME+UVB

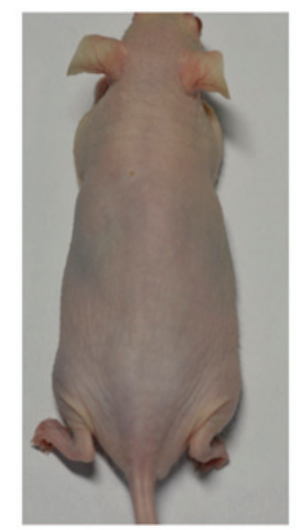

SME+UVB

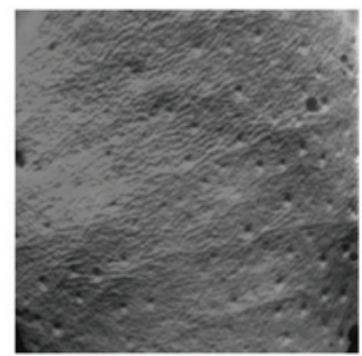

Figure 1. SME prevents UVB-induced wrinkle formation in the dorsal skin of hairless mice. (A) Images of the backs of hairless mice were captured and morphological features of the hairless mouse dorsal skin were recorded using a digital camera. (B) Images of skin replicas were obtained from the dorsal skin of hairless mice following UVB irradiation. SME, ethyl acetate fraction of Sargassum muticum; UVB, ultraviolet B.
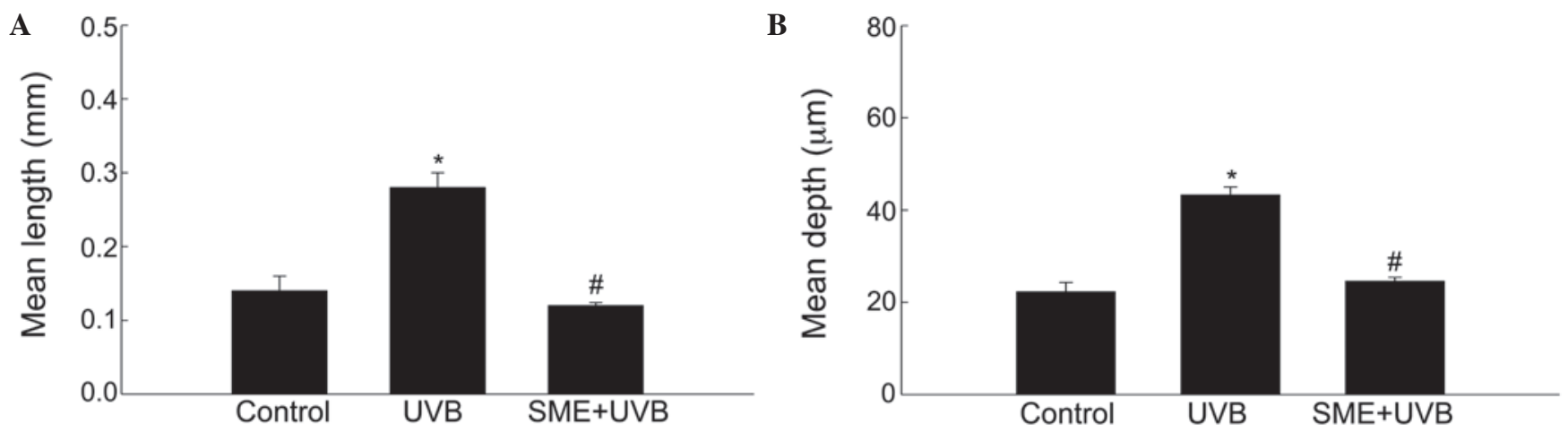

Figure 2. SME mitigates the effects of UVB exposure on the length and depth of hairless mouse skin. The (A) length and (B) depth of skin wrinkles were quantified via analysis of skin replicas using an image analysis system. ${ }^{*} \mathrm{P}<0.05$, vs. untreated control group; ${ }^{*} \mathrm{P}<0.05$, vs. UVB-irradiated group. Values are presented as the mean \pm the standard error of the mean. SME, ethyl acetate fraction of Sargassum muticum; UVB, ultraviolet B.

present study subsequently evaluated changes in the dermal thickness of the skin of UVB-irradiated mice, compared with unexposed control hairless mice. Measurement of the tissue sections indicated that the epidermal thickness of the dorsal skin was significantly increased following UVB irradiation in vehicle-pretreated mice. By contrast, epidermal hypertrophy was significantly reduced by SME pretreatment (Fig. 3A). Additionally, histological observations of hematoxylin and eosin-stained sections revealed that the epidermal thickness in the UVB-irradiated dorsal skin of the vehicle group was markedly increased. However, SME pretreatment inhibited the increase in epidermal thickness (Fig. 3B). The tissue sections were then subjected to Masson's trichrome staining to visualize changes in collagen fiber formation in the dermal areas of the UVB-exposed dorsal skin. Notably, the collagen fibers (blue) in the UVB-treated/SME-pretreated skin showed lower levels of damage and an increased extent of bundle formation, compared with those in the UVB-treated/vehicle-pretreated skin (Fig. 3C).

SME inhibits the UVB-induced increase in the expression and activity of MMP-1 in HaCaT keratinocytes. The cultured HaCaT keratinocytes were exposed to UVB radiation at a dose of $30 \mathrm{~mJ} / \mathrm{cm}^{2}$, and the protein expression level of 
A

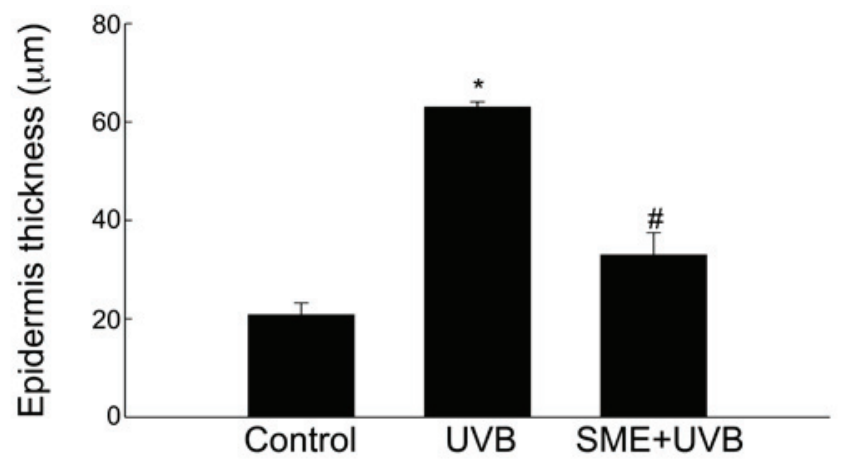

B

Control

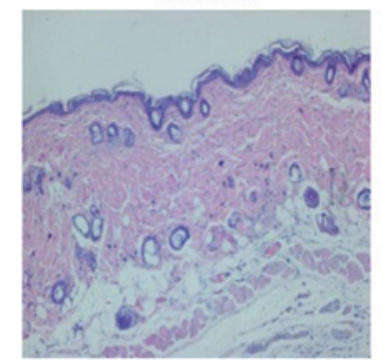

C

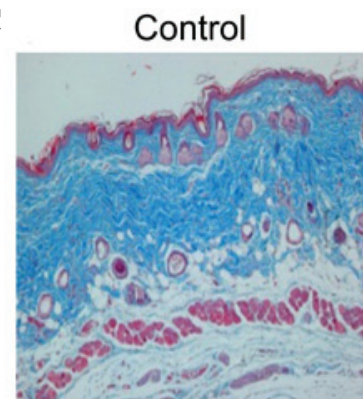

UVB

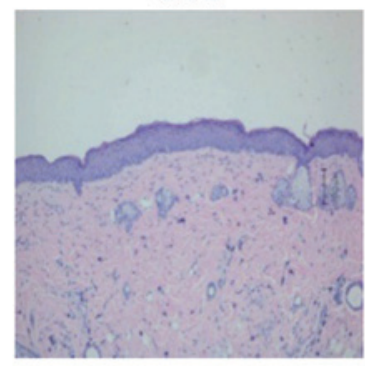

UVB

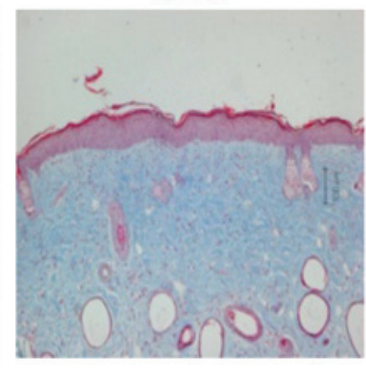

SME+UVB

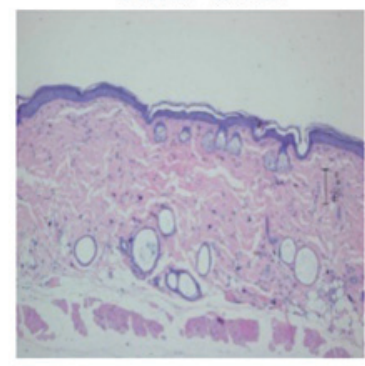

SME+UVB

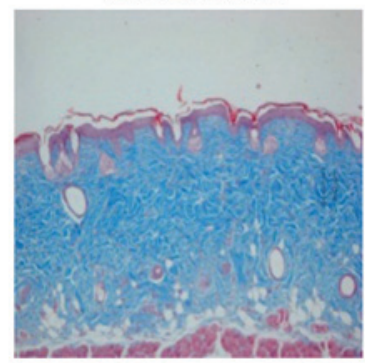

Figure 3. SME inhibits the UVB-induced increase in epidermal thickness in hairless mice. (A) Epidermis thickness was measured under light microscopy with an eyepiece micrometer. Values are presented as the mean \pm the standard error of the mean. ${ }^{*} \mathrm{P}<0.05$, vs. untreated control group and ${ }^{\#} \mathrm{P}<0.05$, vs. UVB-irradiated group. (B) Hairless mouse skin was stained with hematoxylin and eosin (original magnification, x200). (C) Collagen fiber formation was evaluated using Masson's trichrome staining. Collagen fiber bundles are shown in blue (original magnification, x200). SME, ethyl acetate fraction of Sargassum muticum; UVB, ultraviolet B.

MMP-1 was measured using western blot analysis. As shown in Fig. 4A, SME pretreatment inhibited the accumulation of UVB-induced MMP-1 protein $24 \mathrm{~h}$ following exposure. Furthermore, the activity of MMP-1 was significantly enhanced by UVB radiation, but reduced by SME pretreatment (Fig. 4B).

The MMP-1 promoter contains a binding site for the AP-1 transcription factor, and activation of AP-1 by UVB exposure increases the expression of MMP-1 (17). However, SME pretreatment inhibited UVB-induced binding of the AP-1 family member, c-Jun, to the MMP-1 promoter, as determined using a ChIP assay (Fig. 4C). Furthermore, UVB treatment markedly stimulated the expression of phospho-c-Jun, whereas SME treatment decreased its expression (Fig. 4D).

\section{Discussion}

Photoprotection refers to a group of mechanisms developed over the course of evolution to minimize the oxidative damage that may otherwise occur to organs, including the skin, when exposed to UV radiation. The skin is a complex organ composed of multiple layers of tissues and cell types, and serves as a defensive barrier between the internal organs and external factors, particularly UV light. Photoprotective mechanisms in the skin can be stimulated or regulated by naturally occurring compounds or substances originating from terrestrial and aquatic sources. A number of photoprotective compounds derived from assorted marine sources have been developed to prevent photodamage to skin tissues and cells (18). However, the source and safety of natural bioactive molecules possessing anti-photoaging properties has been a major biological concern in the investigation of photoprotection, culminating in the identification of safer marine sources over the past few decades (19). For example, marine brown algae contain non-cytotoxic phenolic compounds, termed phlorotannins, with antioxidant activity and the ability to absorb significant quantities of UV light (20). 
A

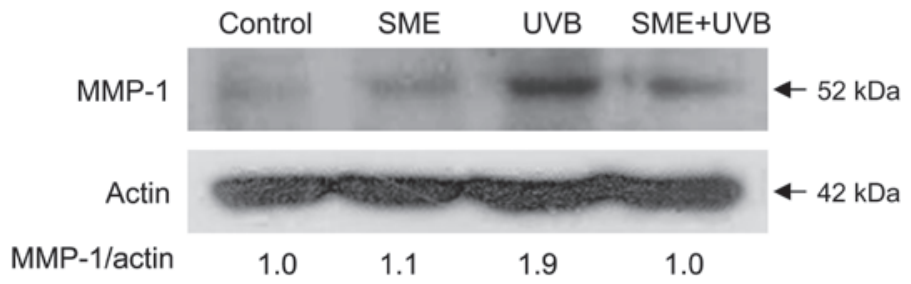

B
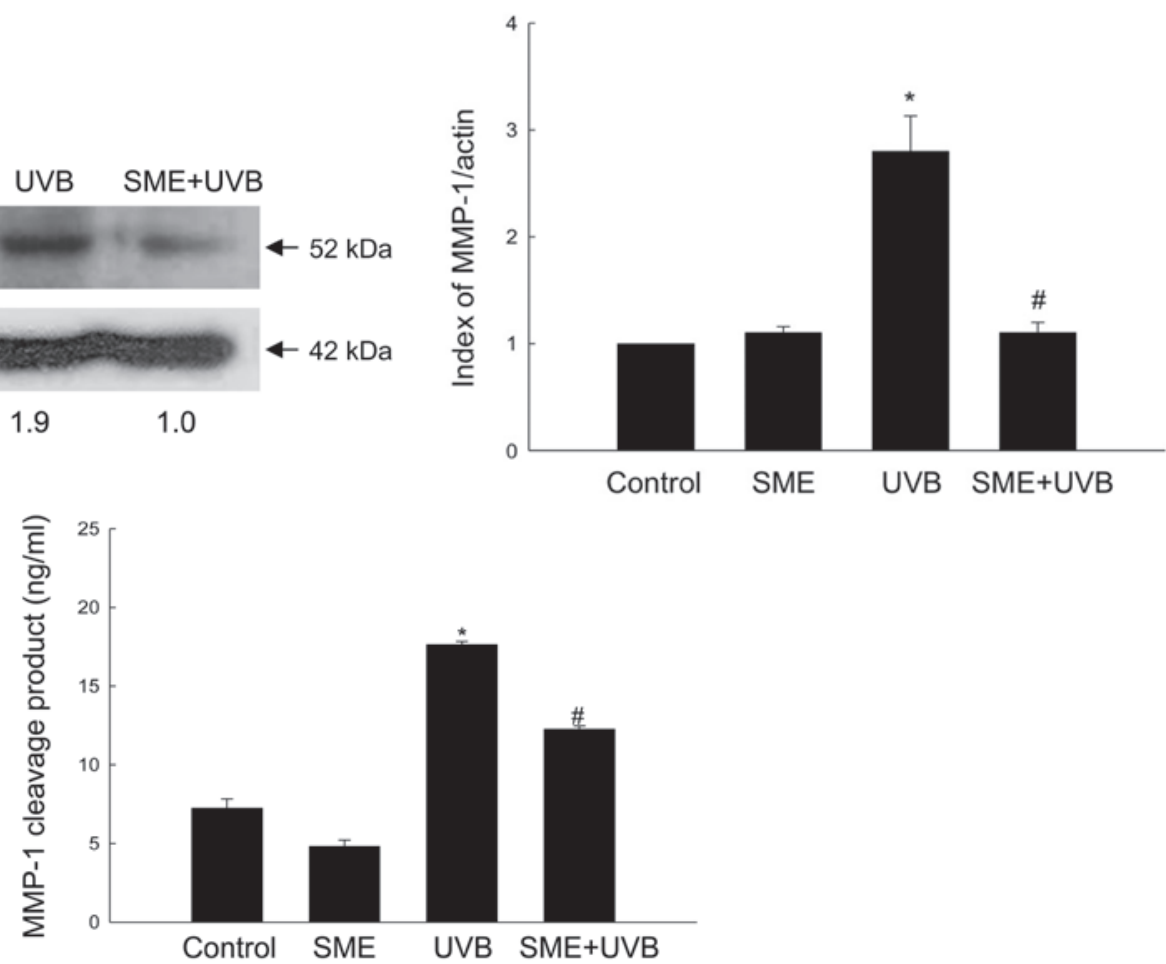

C

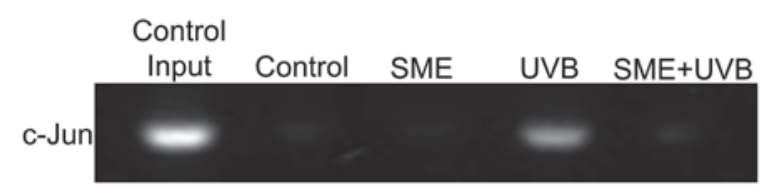

D
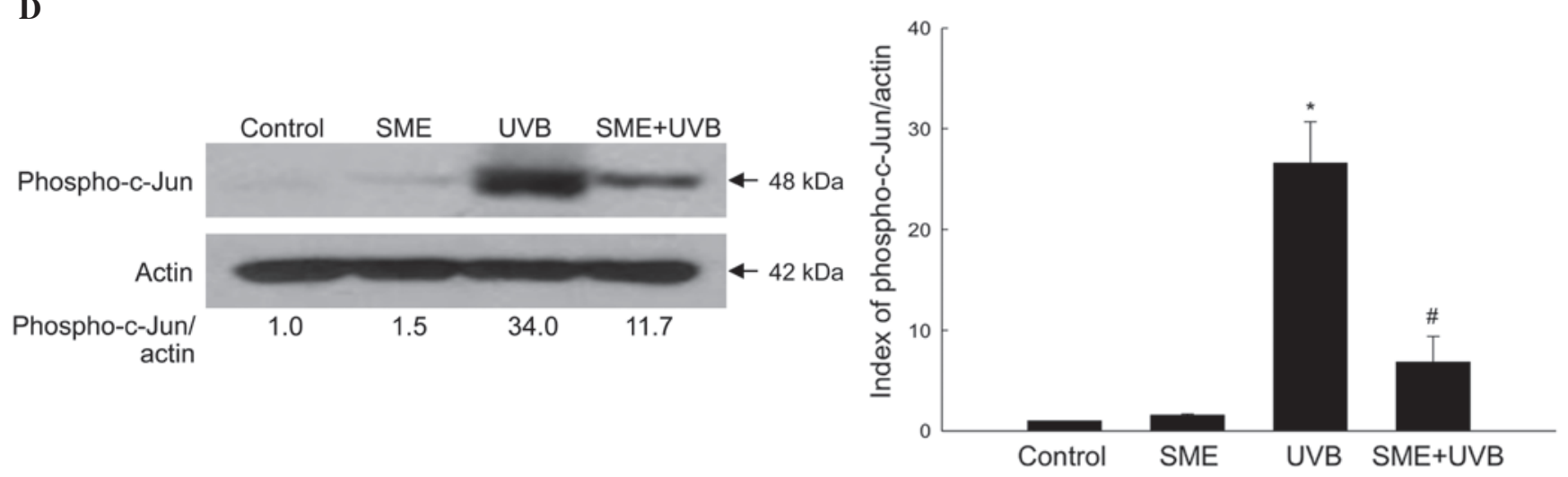

Figure 4. SME protects against the UVB-induced increased expression and activity of MMP-1 in HaCaT keratinocytes. The keratinocytes were irradiated with UVB at a dose of $30 \mathrm{~mJ} / \mathrm{cm}^{2}$ and harvested $24 \mathrm{~h}$ later. (A) Protein expression of MMP-1 was analyzed using western blot analysis and quantified. Actin was used as a loading control. (B) Activity of MMP-1 was determined using a fluorescent assay kit to detect active MMP-1. "P $<0.05$, vs. untreated control cells; ${ }^{\text {"}} \mathrm{P}<0.05$, vs. UVB-irradiated cells. (C) A ChIP assay was performed to detect AP-1 binding to the MMP-1 promoter. (D) Protein expression of phospho-c-Jun was analyzed using western blot analysis and quantified. Actin was used as a loading control. ${ }^{*} \mathrm{P}<0.05$ vs. control cells, ${ }^{\text {}} \mathrm{P}<0.05$ vs. UVB-irradiated cells. SME, ethyl acetate fraction of Sargassum muticum; MMP, matrix metalloproteinase; UVB, ultraviolet B; phospho, phosphorylated.

The results of numerous investigations performed over several years has established the family of marine algae as a principal source of bioactive substances of significant medicinal and nutritional value (21-23). Marine algae reportedly contain several important secondary metabolites, including phenols and polyphenols, resulting from unique linkages known as phenolic couplings (24). These secondary metabolites have a wide array of skeletal backbones and biological activities (19). Although exposure of marine algae to adverse environmental conditions, for example excessive light and high oxygen concentrations, leads to the formation of free radicals and other oxidizing molecules, these conditions do not cause any serious photodynamic damage to the organisms (25). This suggests that marine algae, similar to photosynthesizing plants, possess potent antioxidant mechanisms in addition to compounds that act as antioxidant agents (26).

Brown algae are a staple of Korean and Japanese diets, and their use in traditional Chinese medicine has been documented 
for $>1,000$ years (27). As mentioned above, brown algae contain numerous antioxidant phenolic compounds with the ability to absorb UV light and, therefore, they have been extensively investigated for their anti-photoaging qualities (28). The oral administration of algae-derived dietary antioxidants may protect against chronic UVB-irradiated skin disorders. In this regard, phytochemicals, including proanthocyanidin, genistein and daidzein, are potential photoprotective agents against UVB-induced skin damage (29), and polyphenols and phlorotannins isolated from brown algae exert substantial antioxidant and anti-inflammatory actions (30). The antioxidant activity of these and other compounds associated with $\mathrm{UV}$ protection is currently a focus of investigations due to the increasing demand from the cosmeceutical market and the pharmaceutical industry for efficacious anti-aging products.

Exposure to UV light triggers photoaging of the skin, which is characterized by distinct changes in the components of the dermal connective tissue extracellular matrix, leading to wrinkles, laxity/looseness, coarseness, lentigines, pigmentation, collagen damage and connective tissue modifications (31). Histological studies have revealed that photodamaged skin is also associated with increased epidermal thickness (32). The dermal connective tissue extracellular matrix contains structural proteins (collagen, keratin and elastin), specialized proteins (fibrillin, fibronectin and laminin), glycoproteins and proteoglycans. Disruption of this balanced combination of components has detrimental consequences for skin integrity. Collagen, one of the primary components of skin tissue, is essential for structural maintenance and comprises $70-80 \%$ of the dry weight of the skin. At present, $>20$ types of collagen have been reported (33). Abnormal changes in skin collagen metabolism and decreased collagen levels following excessive UV exposure are largely responsible for skin photoaging and wrinkle formation. However, the present study showed that collagen fibers were restored in UVB-treated/ SME-pretreated skin, compared with the damaged skin of the UVB-treated/vehicle-pretreated mice.

MMP-1 is a critical member of the collagenase subfamily of MMPs, and is the most important interstitial collagenase in humans (34). In the present study, UVB light stimulated the protein expression and activity of MMP-1 in HaCaT keratinocytes, and the binding of the AP-1 transcription factor to the MMP-1 promoter. However, treatment of the cells with SME reversed all the deleterious effects of UVB exposure.

Considering the correlation between anti-photoaging and antioxidant activity, the present study hypothesized that polyphenols and phlorotannins may be central to the protective effect of SME against UVB-induced skin damage. However, additional types of SME-derived compounds may also confer anti-photoaging properties. The precise mechanism by which these compounds potentially safeguard the skin against photoaging remains to be fully elucidated, as SME contains several naturally occurring chemicals, and their concentrations change according to region, climate, season and other exogenous factors. Accordingly, further investigations are required regarding the isolation of active components from SME, and the combinatorial effect of various chemical constituents of the extract.

In conclusion, the present study evaluated the anti-photoaging capacity of SME, and its ability to prevent UVB-induced skin and cell damage in HR-1 strain hairless mice and $\mathrm{HaCaT}$ keratinocytes, respectively. Increased wrinkle formation was observed following the UVB irradiation of hairless mice, however, the oral administration of SME protected against skin aging and injury. Furthermore, SME inhibited UVB-induced increases in epidermal thickness, wrinkle length and depth, and collagen fiber loss in hairless mice. SME also inhibited the UVB-induced upregulated protein expression and activity of MMP-1 in cultured keratinocytes. Based on these results, it was concluded that SME may be a suitable candidate as a photoprotective agent with cosmeceutical and pharmaceutical value.

\section{Acknowledgements}

This study was supported by grants (grant nos. C10010 and K13102) from the Korea Institute of Oriental Medicine and by the National Research Foundation of Korea Grant funded by the Korean Government (Ministry of Education, Science and Technology; grant no. NRF-C1ABA001-2011-0021037).

\section{References}

1. Avila Acevedo JG,Espinosa González AM, De Maria y Campos DM, Benitez Flores Jdel C, Hernandez Delgado T, Flores Maya S, Campos Contreras J, Muñoz López JL and García Bores AM: Photoprotection of Buddleja cordata extract against UVB-induced skin damage in SKH-1 hairless mice. BMC Complement Altern Med 14: 281, 2014.

2. Hwang E, Park SY, Lee HJ, Lee TY, Sun ZW and Yi TH: Gallic acid regulates skin photoaging in UVB-exposed fibroblast and hairless mice. Phytother Res 28: 1778-1788, 2014.

3. Kim JE, Song D, Kim J, Choi J, Kim JR, Yoon HS, Bae JS, Han M, Lee S, Hong JS, et al: Oral supplementation with cocoa extract reduces UVB-induced wrinkles in hairless mouse skin. J Invest Dermatol 136: 1012-1021, 2016.

4. Petrova A, Davids LM, Rautenbach F and Marnewick JL: Photoprotection by honeybush extracts, hesperidin and mangiferin against UVB-induced skin damage in SKH-1 mice. J Photochem Photobiol B 103: 126-139, 2011.

5. Mittal A, Elmets CA and Katiyar SK: Dietary feeding of proanthocyanidin from grape seeds prevents photocarcinogenesis in SKH-1 hariless mice: Relationship to decreased fat and lid peroxidation. Carcinogenesis 24: 1379-1388, 2003.

6. Vayalil PK, Mittal A, Hara Y, Elmets CA and Katiyar SK: Green tea polyphenols prevent ultraviolet light-induced oxidative damage and matrix metalloproteinases expression in mouse skin. J Invest Dermatol 122: 1480-1487, 2004.

7. Yasui H, Hakozaki T, Date A, Yoshii T and Sakurai H: Real-time chemiluminescent imaging and detection of reactive oxygen species generated in the UVB-exposed human skin equivalent model. Biochem Biophys Res Commun 347: 83-88, 2006.

8. Morena M, Delbosc S, Dupuy AM, Canaud B and Cristol JP: Overproduction of reactive oxygen species in end-stage renal disease patients: A potential component of hemodialysis-associated inflammation. Hemodial Int 9: 37-46, 2005.

9. Pashkow FJ: Oxidative sress and inflammation in heart disease: Do antioxidants have a role in treatment and/or prevention? Int J Inflam 2011: 514623, 2011.

10. Zhang N, Andresen BT and Zhang C: Inflammation and reactive oxygen species in cardiovascular disease. World J Cardiol 2: 408-410, 2010.

11. Hong YF, Lee HY, Jung BJ, Jang S, Chung DK and Kim H: Lipoteichoic acid isolated from Lactobacillus plantarum down-regulates UV-induced MMP-1 expression and up-regulates type I procollagen through the inhibition of reactive oxygen species generation. Mol Immunol 67: 248-255, 2015.

12. Chen L, Hu JY and Wang SQ: The role of antioxidants in photoprotection: A critical review. J Am Acad Dermatol 67: 1013-1024, 2012.

13. Kim JY, Lee JA, Kim KN, Yoon WJ, Lee WJ and Park SY: Antioxidative and antimicrobial activities of Sargassum muticum extracts. J Korean Soc Food Sci Nutr 36: 663-669, 2007. 
14. Yoon WJ, Ham YM, Lee WJ, Lee NH and Hyun CG: Brown alga Sargassum muticum inhibits proinflammatory cytokines, iNOS and COX-2 expression in macrophage RAW 264.7 cells. Turk J Biol 34: 25-34, 2010.

15. Piao MJ, Yoon WJ, Kang HK, Yoo ES, Koh YS, Kim DS, Lee NH and Hyun JW: Protective effect of the ethyl acetate fraction of Sargassum muticum against ultraviolet B-irradiated damage in human keratinocytes. Int J Mol Sci 12: 8146-8160, 2011.

16. Gilchrest BA: A review of skin ageing and its medical therapy. $\mathrm{Br}$ J Dermatol 135: 867-875, 1996.

17. Goffin L, Seguin-Estévez Q, Alvarez M, Reith W and Chizzolini C: Transcriptional regulation of matrix metalloproteinase-1 and collagen 1A2 explains the anti-fibrotic effect exerted by proteasome inhibition in human dermal fibroblasts. Arthritis Res Ther 12: R73, 2010.

18. SinhaRP,KlischM,Gröniger A and HäderDP: Ultraviolet-absorbing/ screening substances in cyanobacteria, phytoplankton and macroalgae. J Photochem Photobiol B 47: 83-94, 1998.

19. Pallela R, Na-Young Y and Kim SK: Anti-photoaging and photoprotective compounds derived from marine organisms. Mar Drugs 8: 1189-1202, 2010.

20. Thomas NV and Kim SK: Potential pharmacological applications of polyphenolic derivatives from marine brown algae. Environ Toxicol Pharmacol 32: 325-335, 2011.

21. Abdul QA, Choi RJ, Jung HA and Choi JS: Health benefit of fucosterol from marine algae: A review. J Sci Food Agric 96: 1856-1866, 2016.

22. Fan X, Bai L, Zhu L, Yang L and Zhang X: Marine algae-derived bioactive peptides for human nutrition and health. J Agric Food Chem 62: 9211-9222, 2014.

23. Kim SK and Karagozlu MZ: Marine algae: natural product source for gastrointestinal cancer treatment. Adv Food Nutr Res 64: 225-233, 2011.

24. Torres MA, Barros MP, Campos SC, Pinto E, Rajamani S, Sayre RT and Colepicolo P: Biochemical biomarkers in algae and marine pollution: A review. Ecotoxicol Environ Saf 71: 1-15, 2008.
25. Jiménez-Escrig A, Jiménez-Jiménez I, Pulido R and Saura-Calixto F: Antioxidant activity of fresh and processed edible seaweeds. J Sci Food Agric 81: 530-534, 2001.

26. Heo SJ, Ko SC, Cha SH, Kang DH, Park HS, Choi YU, Kim D, Jung WK and Jeon YJ: Effect of phlorotannins isolated from Ecklonia cava on melanogenesis and their protective effect against photo-oxidative stress induced by UV-B radiation. Toxicol In Vitro 23: 1123-1130, 2009.

27. McLellan DS and Jurd KM: Anticoagulants from marine algae. Blood Coagul Fibrinolysis 3: 69-77, 1992.

28. Henry BE and Alstyne KLV: Effects of UV radiation on growth and phlorotannins in Focus gardneri (phaeophyceae) juveniles and embryos. J Phycol 40: 527-533, 2004.

29. Kang TH, Park HM, Kim YB, Kim H, Kim N, Do JH, Kang C, Cho Y and Kim SY: Effects of red ginseng extract on UVB irradiation-induced skin aging in hairless mice. J Ethnopharmacol 123: 446-451, 2009.

30. Ananthi S, Raghavendran HR, Sunil AG, Gayathri V, Ramakrishnan $G$ and Vasanthi HR: In vitro antioxidant and in vivo anti-inflammatory potential of crude polysaccharide from Turbinaria ornata (Marine Brown Alga). Food Chem Toxicol 48: 187-192, 2010.

31. Kondo S: The roles of cytokines in photoaging. J Dermatol Sci 23 (Suppl 1): S30-S36, 2000.

32. Rittié L and Fisher GJ: UV-light-induced signal cascades and skin aging. Ageing Res 1: 705-720, 2002.

33. Son ED, Choi GH, Kim H, Lee B, Chang IS and Hwang JS: Alpha-ketoglutarate stimulates procollagen production in cultured human dermal fibroblasts, and decreases UVB-induced wrinkle formation following topical application on the dorsal skin of hairless mice. Biol Pharm Bull 30: 1395-1399, 2007.

34. Ryoo IJ, Moon EY, Kim YH, Lee IS, Choo SJ, Bae KH and Yoo ID: Anti-Skin Aging effect of Syriacusins from Hibiscus syriacus on ultraviolet-irradiated human dermal fibroblast cells. Biomol Ther 18: 300-307, 2010. 